IP Periodica Polytechnica Chemical Engineering

62(2), pp. 156-166, 2018

https://doi.org/10.3311/PPch.10861

Creative Commons Attribution (i)

REVIEW ARTICLE

\section{Bio-based 3-hydroxypropionic Acid: A Review}

\author{
Aladár Vidra ${ }^{1}$, Áron Németh ${ }^{1 *}$
}

Received 06 April 2017; accepted after revision 28 July 2017

\begin{abstract}
3-hydroxypropionic acid is a commercially valuable, important platform chemical. It can serve as a precursor for several key compound, such as acrylic acid, 1,3-propanediol, methyl acrylate, acrylamide, ethyl 3-HP, malonic acid, propiolactone and acrylonitrile. Several microorganisms can produce through a range of metabolic pathways. It is indispensable for the commercial production of 3-HP to use cheap and abundant substrates and also to produce in highly efficient processes which could result high yield, titer and productivity. Because of the fact, that natural microorganism do not perform these conditions, metabolic engineering and genetically engineered microorganism are widely used for research and production as well. Several metabolic pathways are introduced to utilize glucose or glycerol for 3-HP production. In this overview naturally producer microorganisms, synthetic biochemical pathways, results from the recent years and recovery of 3-HP are detailed.
\end{abstract}

\section{Keywords}

3-hydroxypropionic acid, 3-HP, biochemical pathway, yield, titer, productivity, recovery
${ }^{1}$ Department of Applied Biotechnology and Food Science,

Faculty of Chemical Technology and Biotechnology,

Budapest University of Technology and Economics,

Budafoki út 6-8., H-1111 Budapest, Hungary

${ }^{*}$ Corresponding author, e-mail: naron@f-labor.mkt.bme.hu

\section{Introduction}

3-Hydroxypropionic acid (3-HP) is a commercially valuable, important platform chemical which is one of the 12 platform chemicals selected by the US Department of Energy that can be derived from biomass $[1,2]$. It is a 3-carbon, non-chiral organic molecule and a structural isomer of lactic acid. It naturally exists in a small number of thermophilic archaea and bacteria, furthermore participates in autotrophic carbon fixation cycles [3]. The two functional groups, a carboxyl group and a $\beta$ hydroxyl group give high reactivity for 3-HP and they also make it a suitable precursor for synthesizing many optically active substances. While in chemical industries 3-HP is used as crosslinking agent for polymer coatings, metal lubricants and antistatic agents for textiles, it can also be used as surgical biocomposite material and drug release material [4]. The 3-HP can serve as a precursor for several key compounds, such as acrylic acid, 1,3-propanediol, methyl acrylate, acrylamide, ethyl 3-HP, malonic acid, propiolactone and acrylonitrile [5].

Several chemical synthesis routes have been described to produce 3-HP but the costs of these routes are too high because of the expensive starting materials and the high operation costs as well as environmental incompatibility. These resulted limited application area and the absence of 3-HP as a bulk chemical. Compared with chemical routes biological routes from renewable resources have some advantages [6]:

- Relieve the dependence on ever more expensive petroleum and improve energy security.

- Less or no net contribution of carbon dioxide to the atmosphere.

- Less environmental pollution

- More mild operation conditions.

\section{3-HP biosynthesis}

There are several microorganisms in nature that have incorporated 3-HP routes in their biochemical pathways or can convert renewable resources (such as glycerol or glucose) to 3-HP under specific growth conditions. Therefore, it is important to understand the metabolic network of the natural producers in order to increase 3-HP formation when growing on renewable resources [7]. 


\subsection{Natural 3-HP producers}

\subsubsection{3-HP production from glycerol}

Some Lactobacillus species can produce $3-\mathrm{HP}$ and 1,3-propanediol from glycerol. This production is a twostep reaction. The first step is the conversion of glycerol to 3-hydroxypropionaldehyde by glycerol dehydratase (EC: 4.2.1.30). The next step is the 3-hydroxypropionaldehyde conversion to equimolar quantities of 3-HP and 1,3-propanediol by an aldehydic disproportion of 3-hydroxypropionaldehyde, particularly when glycerol fermentation proceeded in the absence of sugar $[2,6,8]$.

\subsubsection{3-HP production from acrylic acid}

While acrylic acid is one of the targeted compounds of 3-HP platform a range of microorganisms can reconvert acrylic acid to 3-HP. It is reported in Byssochlamys sp. and also observed the 3-HP accumulation during the degradation of acrylic acid by Geotrichum sp. and Trichoderma sp. The acrylic acid was metabolized to 3-HP by Alcaligenes faecalis and Rhodococcus erythropolis, as well $[9,10]$.

\subsubsection{3-HP production from $\mathrm{CO}_{2}$}

A different group of microorganisms that has reported to produce 3-HP, can possesses the autotrophic carbon dioxide assimilation pathway, known as the 3-hydroxypropionate $\mathrm{CO}_{2}$ bi-cycle (Fig. 1). Chloroflexus aurantiacus, a thermophilic photosynthetic bacterium, secretes 3-HP during phototrophic growth through the 3-hydroxypropionate cycle. There are several microorganisms that have been reported to own a version of this autotrophic pathway, such as Acidianus brierleyi, Acidianus ambivalens, Sulfolobus metallicus, Metallosphaera sedula, Crenarchaeota and Roseiflexus spp. 3-Hydroxypropionate/4hydroxybutyrate cycle, one of the numerous pathways for $\mathrm{CO}_{2}$ assimilation, was first discovered in Metallosphaera sedula, a model archea for this pathway. In both the pathways, the biotin-dependent acetyl-CoA carboxylase (EC:6.4.1.2) and propionyl-CoA carboxylase (EC:6.2.1.17) are the enzymes that catalyze $\mathrm{CO} 2$ fixation within the cycle (Step 1 and Step 7. on Fig. 1). The first reaction in the pathways are acetyl-CoA carboxylation into malonyl-CoA by acetyl-CoA carboxylase (EC:6.4.1.2) (Step 1. on Fig. 1). This reaction consumes one ATP molecule. The malonyl-CoA reduced to 3-HP through malonate semialdehyde applying two NADPH molecules (Step 2-3. on Fig. 1). 3-HP is first reduced to propionyl-CoA then carboxylated to methylmalonyl-CoA which is converted to succinyl-CoA (Step 4-8. on Fig. 1). Thereafter, the two pathways use different routes $[2,11]$.

3-HP production from $\mathrm{CO}_{2}$ is attractive because $\mathrm{CO} 2$ is readily available and other carbon source comprises a significant part of the manufacturing cost. For industrial application autotrophic 3-HP production by Chloroflexus aurantiacus or the cyclic pathway has not been examined.
However different steps of these pathways were expressed and studied in GMO's (see Section 2.2). [2, 3]

\subsubsection{3-HP production via uracil catabolism pathway}

3-HP was also produced as a degradation product of a pyrimidine base, uracil. A novel uracil catabolism pathway in Saccharomyces kluyveri is independent of the respiratory chain. It was founded that uridine monophosphate and urea were intermediates, and 3-HP, ammonia and carbon dioxide were the final products of degradation in this pathway $[2,8]$.

\subsubsection{3-HP production via unknown mechanism}

Some endophytic fungi can excrete $3-\mathrm{HP}$ as a nematicide. 3-HP was extracted from the culture filtrate of several endophytic fungi isolated from above-ground plant organs. It is observed that 3-HP exhibited selective nematicidal activity against the plant-parasitic nematode Meloidogyne incognita with LD50 (Lethal dose, 50\%) of $12.5-15 \mu \mathrm{g}^{*} \mathrm{ml}^{-1}$, such as Phomopsis phaseoli, Melanconium betulinum, Betula pendula and Betula pubescens. The biochemical pathways for 3-HP production in these fungi have not been elucidated [12].

It is indispensable for the 3-HP commercial production to use cheap and abundant substrates and also indispensable to utilize in highly efficient production processes that can result in high titer, yield and productivity. The natural microorganism cannot fulfill these criteria. Therefore metabolic engineering and genetically engineered microorganism are necessary to make the biochemical production of 3-HP commercially available $[2,10]$.

\subsection{Constructed biochemical pathways for 3-HP production}

\subsubsection{Starting from glucose}

For 3-HP production from glucose seven biochemical pathways were developed by Cargill, an agricultural company. The pathways are recommended to be redox-balanced and to generate ATP otherwise it reach the redox-balance with byproduct production which reduces the product yield and increases the byproduct formation. All the seven pathways are redox neutral, but most of them do not produce ATP. Pyruvate and phosphoenolpyruvate (PEP) are important intermediates in these pathways. While one mol glucose is oxidized to two mol pyruvate, two mol NADH and two mol ATP is generated. ATP yield depends on the enzymes in the pathways [6].

In Pathway I (red and yellow route on Fig. 2) one mol ATP per mol 3-HP is produced if the conversion of lactate to lactoyl-CoA is mediated by CoA-transferase (EC:2.8.3.5), which is otherwise an ATP consuming reaction [2].

In Pathway II (brown and orange route on Fig. 2) the conversion of glucose into acetyl-CoA generates one ATP and two NADH per acetyl-CoA formation. On the other hand, the carboxylation of acetyl-CoA to malonyl-CoA utilizes 1 ATP, and the reduction of malonyl-CoA to 3-HP consumes two 


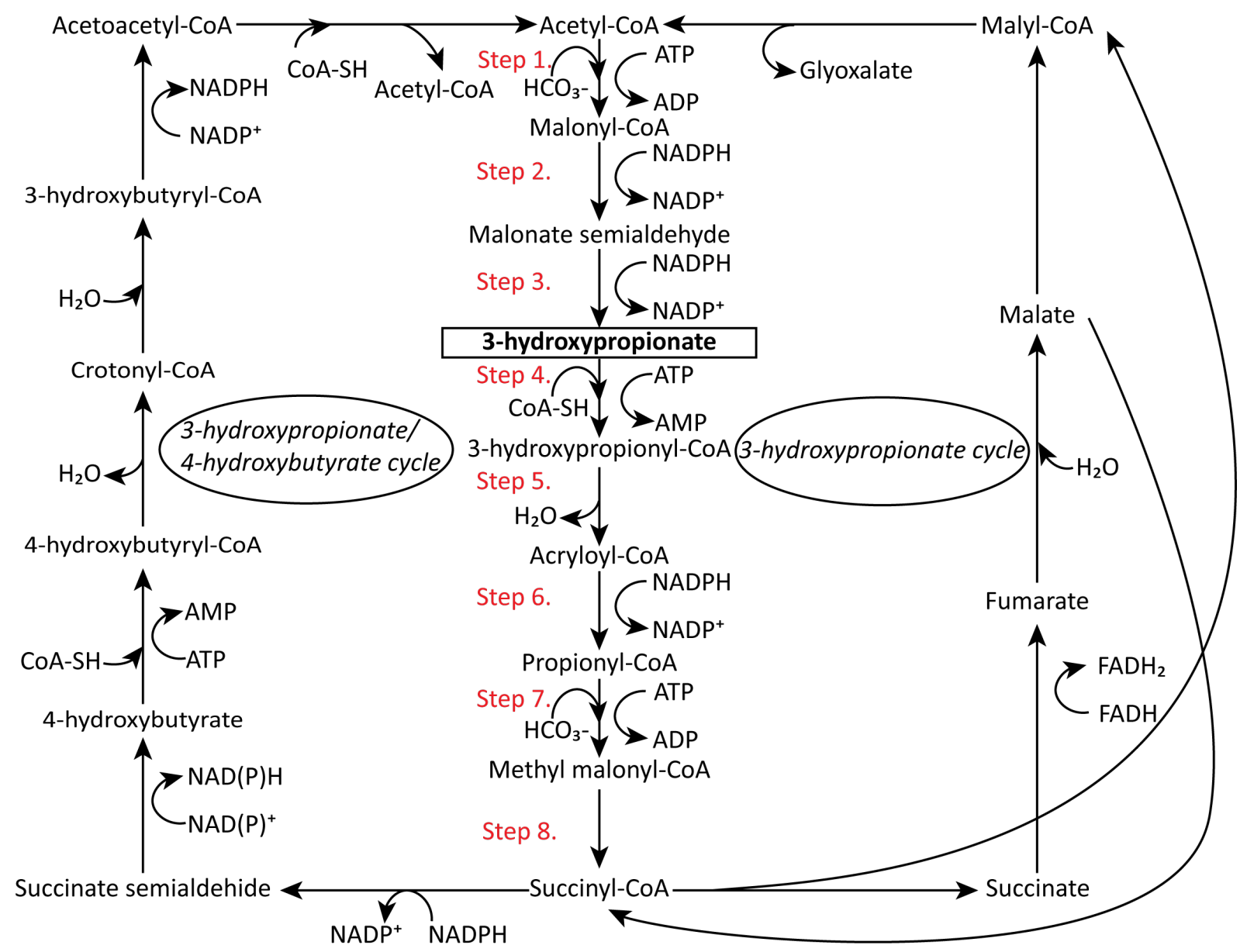

Fig. 1 Production of 3-HP as an intermediate in autotrophic pathways, 3-hydroxypropionate (3-HP) and 3-hydroxypropionate/4-hydroxybutyrate (3-HP/4-HB) cycle. Common steps are numbered. FAD: flavin adenine dinucleotide [2].

NADPH per 3-HP. This pathway is redox neutral but produces no net ATP. In addition to a lack of net ATP generation, the inability of malonyl-CoA reductase(EC:1.2.1.75) to utilize NADH as a cofactor is a significant disadvantage $[3,6]$.

Pathway III (green, purple and orange route on Fig. 2) and Pathway IV (green, light blue and yellow route on Fig. 2) where three steps are in common to the aspartate, involve the carboxylation of pyruvate/PEP to oxaloacetate. The carboxylation of PEP to oxaloacetate produces a single ATP molecule if mediated by PEP carboxykinase (EC:4.1.1.49) and does not generate ATP when catalyzed by PEP carboxylase (EC:4.1.1.31). The ATP yield depends on the intermediate (pyruvate/PEP) and/or the enzyme (PEP carboxylase/carboxykinase) $[6,10]$.

In the case of Pathway V (dark blue and yellow route on Fig. 2), which is based on the succinic acid fermentative route with propionate as an intermediate, ATP production becomes negative. Pyruvate or PEP is converted to succinate, which is transformed to propionyl-CoA directly or through propionate. The dehydrogenation of propionyl-CoA removes two $[\mathrm{H}]$ and gives acryloyl-CoA, which is hydrated to 3-hydroxy propionyl-CoA followed by its hydrolysis to 3-HP. There is net consumption of $1 \mathrm{~mol}$ of ATP per mol of 3-HP produced.
When oxaloacetate is obtained from carboxylation of PEP by PEP carboxykinase (EC:4.1.1.49), the ATP produced and consumed is balanced. However, according to a report by Emde and Schink, the net ATP production becomes -0.33 ATP per 3-HP if propionyl-CoA is produced via propionate [7, 8].

Pathway VI (rose, purple and orange route on Fig. 2) and Pathway VII (rose, light blue and yellow route on Fig. 2) depend on alanine-2,3-aminomutase. Aminomutases are the enzymes that catalyze the transfer of amino group from $\alpha$-to $\beta$-carbon, and have been engineered to be active on L-amino acids. Lysine 2,3-aminomutase (EC:5.4.3.2) of Porphyromonas gingivalis is engineered to convert $\alpha$-alanine to $\beta$-alanine. The ATP yield (per 3-HP) for pathway VI is +1 . For the pathway VII it can be 1 or 0 depending on the conversion of $\beta$-alanine to $\beta$-alanyl-CoA conversion [13].

Four of these pathways are thermodynamically unfavorable (I, IV, V and VII). Among the ATP producing pathway, Pathway VI is thermodynamically favorable. It is considered the most promising among the seven pathways. In Pathway VI, although the conversion of $\beta$-alanine to 3-oxopropanoate is thermodynamically unfavorable, it can be overcome using a high substrate concentration and low product concentration. 
Table 1 The ATP yield and thermodynamic feasibility for various metabolic pathways starting with glucose for the production of 3-HP. Net ATP yield depend on the enzyme used in the pathways. OAA: Oxaloacetate, PEP: phosphoenolpyruvate. [2]

\begin{tabular}{|c|c|c|}
\hline Metabolic pathway & $\begin{array}{l}\text { Net ATP yield } \\
\text { (mol/mol 3-HP) }\end{array}$ & $\begin{array}{l}\text { Thermodynamic } \\
\text { feasibility }\end{array}$ \\
\hline I $($ Pyruvate $\rightarrow$ lactate $\rightarrow$ lactoyl-CoA $\rightarrow$ acryloyl-CoA $\rightarrow 3-\mathrm{HP}-\mathrm{CoA} \longrightarrow 3-\mathrm{HP})$ & $1 / 0$ & Unfavorable \\
\hline II (Pyruvate $\rightarrow$ acetyl-CoA $\rightarrow$ malonyl-CoA $\rightarrow 3$-oxopropanoate $\rightarrow 3$-HP) & 0 & Favorable \\
\hline III (Pyruvate/PEP $\rightarrow$ OAA $\rightarrow$ aspartate $\rightarrow \beta$-alanine $\rightarrow 3$-oxopropanoate $\rightarrow 3$-HP) & $1 / 0$ & Favorable \\
\hline IV $($ Pyruvate $/ \mathrm{PEP} \rightarrow \mathrm{OAA} \rightarrow$ aspartate $\rightarrow \beta$-alanine $\rightarrow \beta$-alanyl-CoA $\rightarrow$ acryloyl-CoA $\rightarrow 3$-HP-CoA $\rightarrow 3-\mathrm{HP}$ ) & $0 /-1$ & Unfavorable \\
\hline $\mathrm{V}($ Pyruvate $/ \mathrm{PEP} \rightarrow$ succinate $\rightarrow$ propionate/propionyl-CoA $\rightarrow$ acryloyl-CoA $\rightarrow 3-\mathrm{HP}-\mathrm{CoA} \rightarrow 3-\mathrm{HP})$ & $-1 /-0.33$ & Unfavorable \\
\hline VI (Pyruvate $\rightarrow \alpha$-alanine $\rightarrow \beta$-alanine $\rightarrow 3$-oxopropanoate $\rightarrow 3$-HP) & 1 & Favorable \\
\hline VII (Pyruvate $\rightarrow \alpha$-alanine $\rightarrow \beta$-alanine $\rightarrow \beta$-alanyl-CoA $\rightarrow$ acryloyl-CoA $\rightarrow 3$-HP-CoA $\rightarrow 3$-HP) & $1 / 0$ & Unfavorable \\
\hline
\end{tabular}

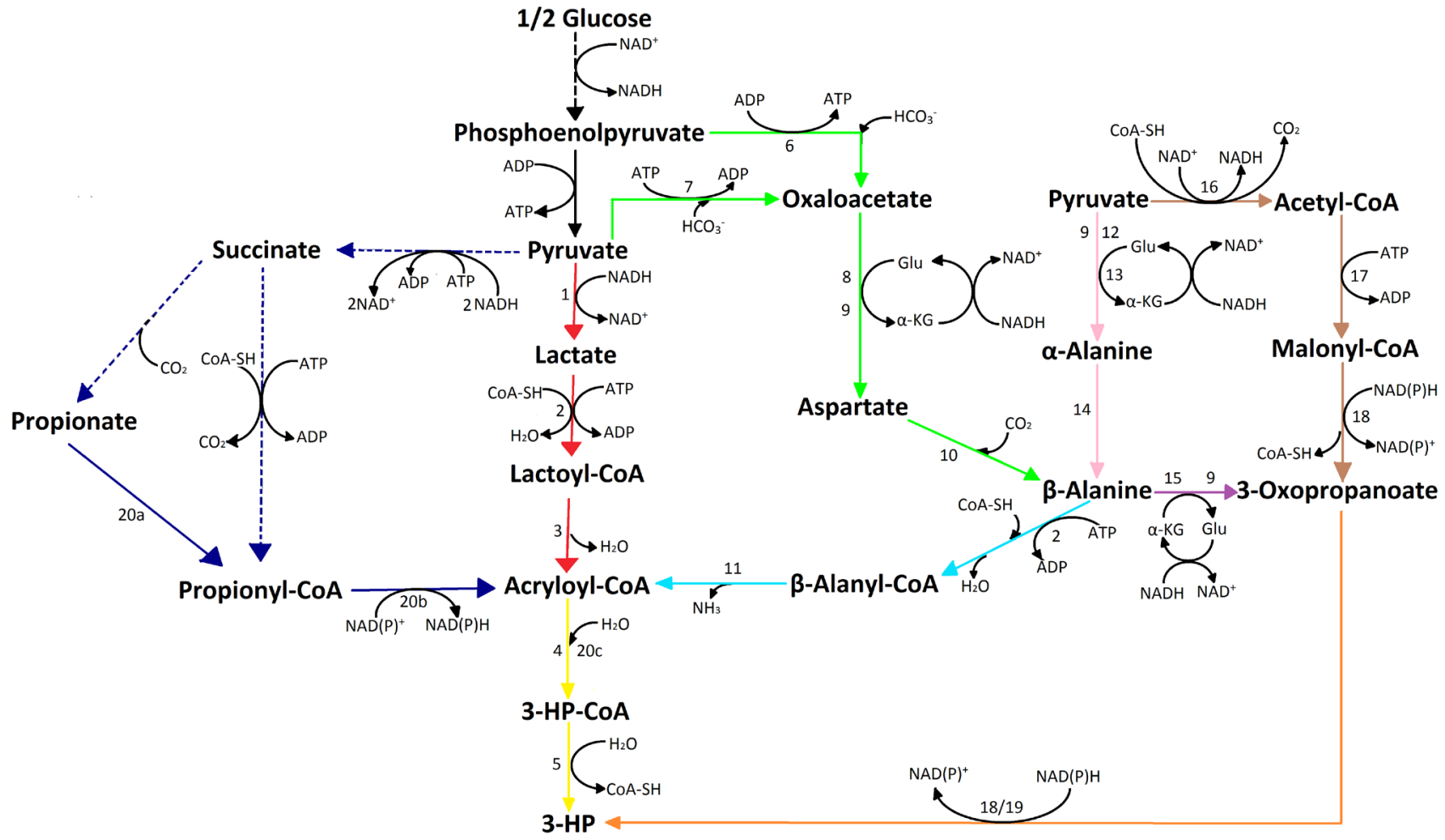

Fig. 2 Biochemical pathways for 3-HP production from glucose. The enzymes involved are as follows: 1, lactate dehydrogenase; 2, acetate-CoA ligase/CoA-transferase; 3, lactyl-CoA dehydratase; 4, 3-HP-CoAdehydratase; 5, 3-HP-CoAhydrolase/CoA-transferase/3-hydroxyisobutrylCoAhydrolase; 6 , Phosphoenolpyruvate carboxylase/carboxykinase; 7 , pyruvate carboxylase; 8 , aspartate aminotransferase; 9 , glutamate dehydrogenase;

10, aspartate decarboxylase; 11, $\beta$-alanine-CoAammonium lyase; 12 , pyruvate-glutamate transaminase; 13, alanine dehydrogenase; 14 , alanine

2,3-aminomutase; 15 , 4-aminobutyrate aminotransferase/ $\beta$-alanine-2-oxoglutarate aminotransferase; 16 , pyruvate dehydrogenase complex; 17 , acetyl-CoA carboxylase 18, malonyl-CoAreductase; 19, 3-hydroxyisobutyrate dehydrogenase; 20, 3-hydroxyisobutyrate dehydrogenase (consists of three functional domains) a-CoA-synthetase, b-dehydrogenase \& c-3-HP dehydratase (Glu: glutamate; $\alpha$-KG: $\alpha$-ketoglutarate) [2]

\subsubsection{Starting from glycerol}

Glycerol, the main byproduct of biodiesel production, is a usable substrate for 3-HP fermentation. It is non-toxic to many microorganisms and it can be used in high concentration. Some microorganisms, such as Klebsiella pneumoniae, Lactobacillus reuteri and Lactobacillus collinoides produce 3-HP by oxidizing 3-hydroxypropionaldehyde (3-HPA). There are two main routes towards the production of 3-HP from glycerol (Fig. 3). that have been investigated during the last years; CoAdependent pathway and CoA-independent pathway $[2,6]$.
The CoA-dependent pathway was based on 1,2-propanediol (1,2-PDO) degradation in Salmonella enterica. All the genes necessary for the consumption of 1,2-PDO by Salmonella enter$i c a$ are found in the propanediol utilization $(p d u)$ locus. The production of 3-HP from glycerol can be carried out through precisely the same pathway as the enzymes used for the production of propionate. In this case, the reaction sequence is as follows: glycerol $\rightarrow 3$-HPA $\rightarrow 3-\mathrm{HP}-\mathrm{CoA} \rightarrow 3$-HP-phosphate $\rightarrow$ 3-HP. Glycerol is converted to 3-HPA by the B12-dependent diol-dehydratase (EC:4.2.1.28). Then the 3-HP is reached 
through 3-HP-CoA and 3-HP-phosphate. The reactions are catalyzed by (CoA)-dependent propionaldehyde dehydrogenase (EC:1.2.1.3) (PduP), phosphotransacylase (EC:2.1.3.222) $(\mathrm{P} d u \mathrm{~L})$ and propionate kinase $(\mathrm{EC}: 2.7 .2 .15)(\mathrm{P} d u \mathrm{~W})$. The last reaction produces one ATP. The same pathway is also present in Klebsiella pneumoniae [8, 10].

In the CoA-independent pathway, the dehydration of glycerol to 3-HPA is catalyzed by glycerol dehydratase (EC:4.2.1.30), and then oxidation of 3HPA to 3HP is catalyzed by aldehyde dehydrogenase(ALDH) (EC:1.2.1.3). Unlike the CoA-dependent pathway, this pathway neither produces ATP nor requires the participation of $\mathrm{CoA}$. This pathway is much simpler than the CoA-dependent pathway and can utilize the multiple ALDHs present in many microorganisms. On the other hand, the functioning of this pathway for 3-HP production in natural microorganisms has not been elucidated [14-17].

In both pathways, aldehyde dehydrogenases require $\mathrm{NAD}^{+}$ as a cofactor, which should be regenerated by the oxidative metabolism of glycerol and/or an electron transport chain.

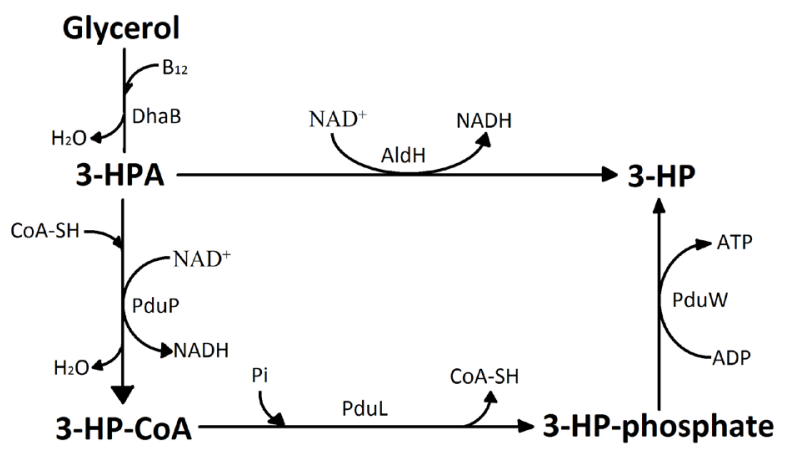

Fig. 3 Simplefied Biochemical reactions showing production of 3-HP from glycerol by CoA-dependent and CoA-independent pathways [2]

\subsubsection{Novel biochemical pathway for 3-HP production suggested by in silico approach}

The Biochemical Network Integrated Computational Explorer (BNICE) software can design pathways with the ability to incorporate novel reactions and intermediates that are unknown or have not been identified. This program generates every possible biochemical reaction route connecting the starting compound(s) and target product. The BNICE based on NetGen, which is an automated network generation method and a set of enzyme reaction rules. These rules based on a manual curation of the enzyme commission (EC) system, which covers much of the known biochemistry. The pathway ranking is based on several criteria. The criteria are the pathway length, thermodynamic feasibility, maximum achievable yield of 3-HP on glucose, and the maximum achievable intracellular concentration [13]. E. coli was used as a model microorganism and anaerobic cultivation with glucose as the sole carbon source was assumed. BNICE confirmed that among the seven pathways suggested by Cargill, Pathway VI was the most promising. The three novel pathways developed by using BNICE involve the conversion of pyruvate to lactate followed by the formation of 3-HP with a theoretical yield of two 3-HP molecules per molecule of glucose consumed [8].

The first novel pathway on Fig. 4 from BNICE involves only two steps (pyruvate $\rightarrow$ lactate $\rightarrow 3-\mathrm{HP}$ ). This pathway has one novel reaction, the conversion of lactate to 3-HP. This step is thermodynamically unfavorable. Therefore, it was suggested to maintain a high intracellular lactate concentration and low 3-HP concentration. The maximum achievable 3-HP intracellular concentration was estimated to be only $1.0 \mathrm{mM}$.

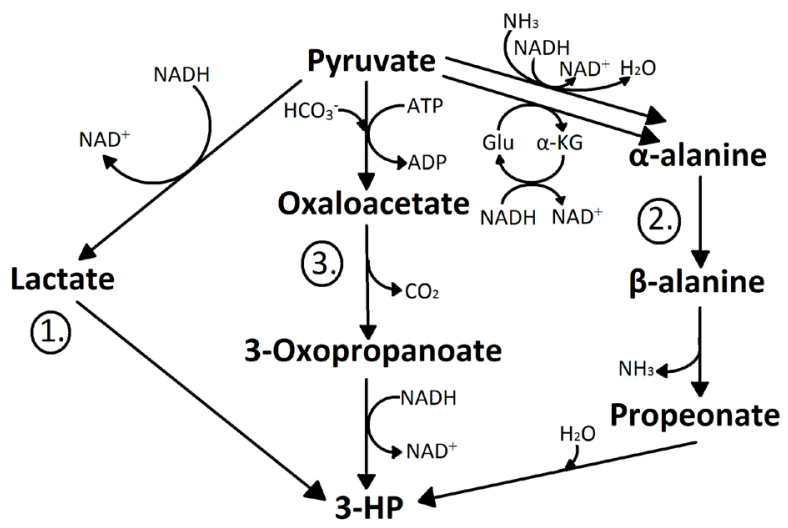

Fig. 4 Simplefied novel pathways generated by BNICE for 3-HP production [2] The different routes are numbered. Glu: glutamate; $\alpha$-KG: $\alpha$-ketoglutarate

The second novel pathway on Fig. 4 (pyruvate $\rightarrow \alpha$-alanine $\rightarrow \beta$-alanine $\rightarrow$ propenoate $\rightarrow 3$-HP) is the same as Cargill's Pathway VI except that only one intermediate, 3-oxopropanoate, is replaced by propenoate. These two pathways differ in terms of the aminating and deaminating reactions involved. The (trans)amination reaction (conversion of pyruvate to $\alpha$-alanine) in these pathways can be carried out by either the incorporation of ammonia or the use of glutamate. The theoretical 3-HP yield in this pathway is $2.0 \mathrm{~mol} / \mathrm{mol}$ glucose, whereas the maximum achievable 3-HP intracellular concentration was estimated to be $8.3 \mathrm{mM}$.

The third pathway on Fig. 4, (pyruvate $\rightarrow$ oxaloacetate $\rightarrow 3$ oxopropanoate $\rightarrow 3-\mathrm{HP}$ ) constructed by BNICE is noteworthy. This pathway is thermodynamically feasible and requires the consumption of one ATP molecule. The theoretical 3-HP yield ( $\mathrm{mol} / \mathrm{mol}$ glucose) is reduced to 1.22 due to the ATP requirement $[2,8]$.

\section{Major challenges for microbial production of 3-HP}

Bioconversion offers a safer and more viable alternative with the opportunity to produce a variety of chemicals under milder conditions. However, there are currently only a very few examples of bio-based processes overcoming the competitive petrol-based process such as yeast-based ethanol, yeast- or lactic acid bacteria-based lactic acid and E. coli-based propanediol. For these bio-based chemicals to be economically feasible, a 
high product concentration (above $100 \mathrm{~g}^{*} \mathrm{~L}^{-1}$ ), productivity (over $2 \mathrm{~g}^{*} \mathrm{~L}^{-1 *} \mathrm{~h}^{-1}$ ) and substrate-to-product yield (above 50\%) are required. Although several biological routes and recombinant strains have been developed for the production of 3-HP, their performance is still unsatisfactory and more improvement is required. Many technical hurdles need to be addressed to realize the biological production of 3-HP. With glucose as a substrate, only few studies have been reported (as shown in Table 2) while there is an interesting patent on high level 3-HP production[18]. Identifying the most significant issues appears to be difficult at this stage. On the other hand, with glycerol as a substrate, detailed information on the relevant genes, enzymes and biochemical pathways are well documented. Therefore, the challenges for commercial 3-HP production are described with particular focus on the latter substrate, glycerol, these will be discussed detailed in the next sections $[2,10]$.

\subsection{Toxicity of 3-HP}

Similar to otheracids, 3-HPcauses $\mathrm{pH}$-based growth inhibition. In most 3-HP production processes with recombinant Klebsiella pneumoniae and Escherichia coli, the rate of production in the late period where the 3-HP concentration exceeds $200-300 \mathrm{mM}$ decreases significantly. In addition, when 3-HP was added to the culture medium, the rates of cell growth, glycerol consumption and 3-HP production decreased progressively as the 3-HP concentration increased. The problems associated with acid toxicity can be solved partly by adapting the microorganisms to low $\mathrm{pH}$ and conducting fermentation at a $\mathrm{pH}$ less than the $\mathrm{pKa}$ of the acid which is $4.51[8,19]$.

\subsection{Toxicity of 3-HPA}

3-hydroxypropionaldehyde (3-HPA), the immediate substrate of 3-HP production, is highly toxic and its accumulation at $15-30 \mathrm{mM}$ often halts 3 -HP production. Although several hypotheses have been proposed for its bactericidal action, the precise mechanism is unclear. 3-HPA accumulation is caused by an imbalance between the activities of glycerol dehydratase (GDHt) (EC:4.2.1.30) and AldH (EC:1.2.1.3) and/or 1,3-propanediol oxidoreductase (PDOR) (EC:1.1.1.202). In addition, propanediol build-up can contribute to the accumulation of 3-HPA because PDOR can catalyze the reverse reaction $(\mathrm{PDO} \rightarrow 3$-HPA) under high propanediol concentrations. During 3-HP production from glycerol, 3-HPA accumulation can be avoided by balancing the rates of its production and consumption. This can be accomplished by either reducing the GDHt activity or enhancing the activity of AldH. The former strategy would reduce the overall rate of production. Therefore, the latter is considered more desirable [10, 20].

\subsection{Vitamin $B_{12}$ supply}

Coenzyme $B_{12}$ is often inactivated during the reaction catalyzed by glycerol dehydratase and should be supplied continuously. In many microorganisms, including K. pneumoniae, synthesis of coenzyme $B_{12}$ is unsatisfactory under aerobic conditions, which are required for the active regeneration of $\mathrm{NAD}^{+}$. Pseudomonas denitrificans produces vitamin $\mathrm{B}_{12}$ under aerobic conditions. The use of $P$. denitrificans as a host might solve the problems associated with aerobic vitamin $B_{12}$ synthesis. On the other hand, the glycerol metabolism has not been reported in P. denitrificans. Furthermore, this organism has not been studied as a recombinant host and no genetic tool box for this strain is available $[2,10]$. Another approach to solve the problem is to use $\mathrm{B}_{12}$-independent glycerol dehydratase from Clostridium butyricum [21, 22].

\subsection{Redox balance and yield}

The regeneration of $\mathrm{NAD}^{+}$is a challenging issue in glycerolbased 3-HP production. The redox balance affects a wide range of genes, cellular functions and metabolite profiles. Determining and maintaining the optimal oxygen level that allow the continuous regeneration of $\mathrm{NAD}^{+}$while not interferes with the synthesis of vitamin $\mathrm{B}_{12}$ and/or proper carbon flux toward 3-HP production is quite difficult. Anaerobic 3-HP production in the presence of nitrate is one strategy to deal with this problem. The potential of this approach was illustrated by the high 3 -HP final titer, $\sim 22 \mathrm{~g}^{*} \mathrm{~L}^{-1}$, in one recombinant $K$. pneumoniae $[8,10]$.

\section{Biotechnological production}

We summarized recent 3-HP production results in Table 2 .

\subsection{Highest 3-HP yield}

Immobilization of Acetobacter sp. CGMCC 8142 was investigated for efficient biocatalysis of 1,3-propanediol to 3-hydroxypropionic acid [23]. The optimal immobilization conditions for 3-HP biocatalysis was sodium alginate of $40 \mathrm{~g}^{*} \mathrm{~L}^{-1}$, gel beads diameter of $1 \mathrm{~mm}$, cross-linkage time $2 \mathrm{~h}$ and $0.1 \mathrm{mM} \mathrm{FeCl2}$. Immobilized cells showed promising substrate tolerance, $\mathrm{pH}$ stability, thermal stability and storability. The bioconversion was prepared in $10 \mathrm{ml}$ final reaction volume. The reaction mixture contained 5.0 g CDW (Cell Dry Weight) pro Liter, $70 \mathrm{~g}^{*} \mathrm{~L}^{-1}$ 1,3-propanediol and acetic acetate buffer (0.2 M, pH 6.0). Gel beads were collected after each cycle and subsequently soaked for $30 \mathrm{~min}$ in saline for removal of residual components. After 5 cycles of reaction, $66.95 \mathrm{~g}^{*} \mathrm{~L}^{-1} 3$-HP was produced from $70 \mathrm{~g}^{*} \mathrm{~L}^{-1} 1,3$-propanediol, which means $0.96 \mathrm{~g}^{*} \mathrm{~g}^{-1}$ yield [23]. Currently, a similar, but larger scale experiment has not been found in literature. 
Table 2 3-HP fermentation results from the recent years

\begin{tabular}{|c|c|c|c|c|c|c|}
\hline Strain & Fermentation, substrate & Scale & Titer & Yield & Productivity & Ref. \\
\hline Acetobacter sp. CGMCC 8142 & $\begin{array}{l}\text { Immobilized cells batch. } \\
\text { 1,3-propanediol }\end{array}$ & $100 \mathrm{~mL}$ flask & 67.0 & 0.96 & 0.90 & {$[23]$} \\
\hline \multirow[t]{2}{*}{ Corynebacterium glutamicum MH15 } & Fed-batch, glucose & $5 \mathrm{~L}$ bioreactor & 62.6 & 0.51 & 0.87 & {$[24]$} \\
\hline & Fed-batch, glucose \& xylose & $5 \mathrm{~L}$ bioreactor & 54.8 & 0.49 & 0.76 & {$[24]$} \\
\hline E.coli BL21_dhaB_dhaR_aldH & Fed-batch, glucose \& glycerol & 2,5 L bioreactor & 14.3 & - & 0.26 & {$[25]$} \\
\hline E.coliSH254_dhaB_KGSADH & Fed-batch, glycerol & $5 \mathrm{~L}$ bioreactor & 38.7 & 0.35 & 0.54 & {$[5]$} \\
\hline E. coli TA2142_aldH_pdu & Batch, glycerol & $250 \mathrm{~mL}$ flask & 5.0 & 0.52 & 0.1 & {$[26]$} \\
\hline E.coli BL21_dhaB_aldH & Fed-batch, glycerol & $5 \mathrm{~L}$ bioreactor & 31.0 & 0.35 & 0.43 & {$[27]$} \\
\hline E. coli SPC006 $\triangle a c k A-p t a, \triangle y q h D, \Delta g l p R$ & Fed-batch, glycerol & $5 \mathrm{~L}$ bioreactor & 42.0 & 0.27 & 1.26 & {$[28]$} \\
\hline $\begin{array}{l}\text { E. coli BL21star DhaB-DhaR PSALDH without } g l p K \\
\text { and } y q h D\end{array}$ & Fed-batch, glycerol & 2,5 L fermentor & 57.3 & 0.88 & 1.59 & {$[15]$} \\
\hline E. coli BL21_dhaB_aldH & Batch, glycerol & $100 \mathrm{~mL}$ flask & 0.58 & 0.47 & 0.02 & {$[4]$} \\
\hline E. coli BL21_mcr_acc_pntAB & Batch, glucose & $250 \mathrm{~mL}$ flask & 0.2 & 0.03 & 0.01 & [29] \\
\hline E. coli JHS01300/pELDRR + pCPaGGRm & Fed-batch, glucose \& xylose & 2,5 L fermentor & 29.4 & 0.36 & 0.54 & {$[30]$} \\
\hline $\begin{array}{l}\text { E. coli W3110 } \triangle a c k A-p t a \triangle y q h D \_d h a B \_g d r A B \text { and } \\
\text { mutant gabD4 }\end{array}$ & Fed-batch, glycerol \& glucose & $5 \mathrm{~L}$ bioreactor & 71.9 & - & 1.8 & {$[20]$} \\
\hline E. coli BL21(DE3) with nitrolase gene (NIT190) & $\begin{array}{l}\text { Repeated batch with immobilized } \\
\text { cells, 3-Hydroxypropionitrile }\end{array}$ & $10 \mathrm{~mL}$ & 184.7 & 0.68 & 36.9 & {$[31]$} \\
\hline E. coli TA3130 & Batch, glycerol & $250 \mathrm{~mL}$ flask & 6.0 & 0.43 & 0.13 & {$[14]$} \\
\hline $\begin{array}{l}\text { E. coli } D F 40 \text { harboring } p U C 19 / K G S A D H \text { and } p D K 7 \\
(p 15 A) / d h a B 123, g d r A B\end{array}$ & Fed-batch, glycerol & $1,5 \mathrm{~L}$ bioreactor & 41.5 & 0.31 & 0.86 & {$[32]$} \\
\hline K. pneumoniae J2B $\triangle$ dhaT_KGSADH & Fed-batch, glycerol & $1,5 \mathrm{~L}$ bioreactor & 16.3 & 0.4 & 0.3 & {$[16]$} \\
\hline K. pneumoniae WM3 pUC18kan $\neg$ aldHec & Fed-batch, glycerol & $5 \mathrm{~L}$ bioreactor & 48.9 & 0.41 & 1.75 & [33] \\
\hline K. pneumoniae $\triangle g l p K \Delta d h a T \_$puиC & Fed-batch, glycerol & $5 \mathrm{~L}$ bioreactor & 22.5 & 0.27 & 0.45 & {$[34]$} \\
\hline K. pneumoniae $\mathrm{Cu}$ & Batch, glycerol & $5 \mathrm{~L}$ bioreactor & 1.9 & 0.1 & 0.08 & {$[35]$} \\
\hline K. pneumoniae J2BA ldhA_KGSADH & Fed-batch, glycerol & 1,5 L bioreactor & 22.7 & 0.34 & 0.38 & {$[36]$} \\
\hline K. pneumoniae $\Delta$ ldh $1 \Delta$ ldh2 2 pta(pTAC-puиC & Fed-batch, glycerol & $5 \mathrm{~L}$ bioreactor & 83.8 & 0.54 & 1.16 & {$[17]$} \\
\hline R. erythropolis $L G 12$ & Batch, acrylic acid & $250 \mathrm{~mL}$ flask & 17.5 & 0.44 & 0.22 & [9] \\
\hline $\begin{array}{l}\text { Synechocystis } \\
\text { Pcpc560-mcr-pntAB-TrbcL_accBCAD-birA }\end{array}$ & Batch fermentation, $\mathrm{CO}_{2}$ & $100 \mathrm{~mL}$ flask & 0.8 & - & 0.006 & {$[37]$} \\
\hline Saccharomyces cerevisiae SCIYC33/pJC5/pJC9 & Batch fermentation, glucose & $100 \mathrm{~mL}$ flask & 0.46 & 0.02 & 0.006 & {$[38]$} \\
\hline $\begin{array}{l}\text { S. cerevisiae ST687 ALD6, SEacsL641P, PDC1, and } \\
\text { TY4-CaMCR-ACC1 }\end{array}$ & Fed batch, glucose & $1 \mathrm{~L}$ bioreactor & 9.8 & 0.33 & 0.098 & {$[1]$} \\
\hline $\begin{array}{l}\text { S. cerevisiae SCE-R2-200 BcBAPAT EcYDFG TcPAND } \\
\text { ScAAT2 ScPYC1/PYC2 }\end{array}$ & Fed-batch, glucose & $500 \mathrm{~mL}$ flask & 13.7 & 0.55 & 0.17 & {$[39]$} \\
\hline Recombinant S. cerevisiae ST2547 & Fed-batch, xylose & 2,7 L bioreactor & 7.37 & 0.37 & 0.06 & {$[19]$} \\
\hline
\end{tabular}

The second highest 3-HP yield in Table 2 is $0.88 \mathrm{~g}^{*} \mathrm{~g}^{-1}$. In this study recombinant Escherichia coli produce 3-HP from glycerol. Double deletion of $g l p \mathrm{~K}$ (glycerol kinase) and $y q h \mathrm{D}$ (alcohol ehydrogenase) directed the glycerol flux to 3-HP biosynthesis and reduced the formation of 1,3-propanediol. Since 3-hydroxypropionaldehyde (3-HPA), a precursor of 3-HP, is toxic to cell growth, the gene encoding Pseudomonas aeruginosa semialdehyde dehydrogenase (PSALDH) (EC:1.2.1.3) highly active on 3-HPA was expressed in E. coli. To produce 3 -HP, a fed-batch culture was performed in a $2.5-\mathrm{L}$ jar fermentor with a 1 -L working volume of defined $\mathrm{R}$ medium at $37^{\circ} \mathrm{C}$ and $\mathrm{pH}$ 6.8. After the depletion of $20 \mathrm{~g}^{*} \mathrm{~L}^{-1}$ glucose initially added, a feeding solution of $800 \mathrm{~g}^{*} \mathrm{~L}^{-1}$ glucose was fed by the $\mathrm{pH}$-stat mode of operation throughout the fed-batch cultivation. At the mid-exponential growth phase (18 h culture time), IPTG (Isopropyl $\beta-\mathrm{D}^{-1}$-thiogalactopyranoside), coenzyme $\mathrm{B}_{12}$ and glycerol were added once at final concentrations of $0.2 \mathrm{mM}$, $20 \mu \mathrm{M}$ and 57-60 $\mathrm{g}^{*} \mathrm{~L}^{-1}$, respectively. The culture temperature was changed to $25^{\circ} \mathrm{C}$ and the jar was covered with aluminum foil. When glycerol was consumed completely, glycerol was added to a final concentration of around $35 \mathrm{~g}^{*} \mathrm{~L}^{-1}$. Finally, fed-batch culture of recombinant $E$. coli BL21star(DE3) 


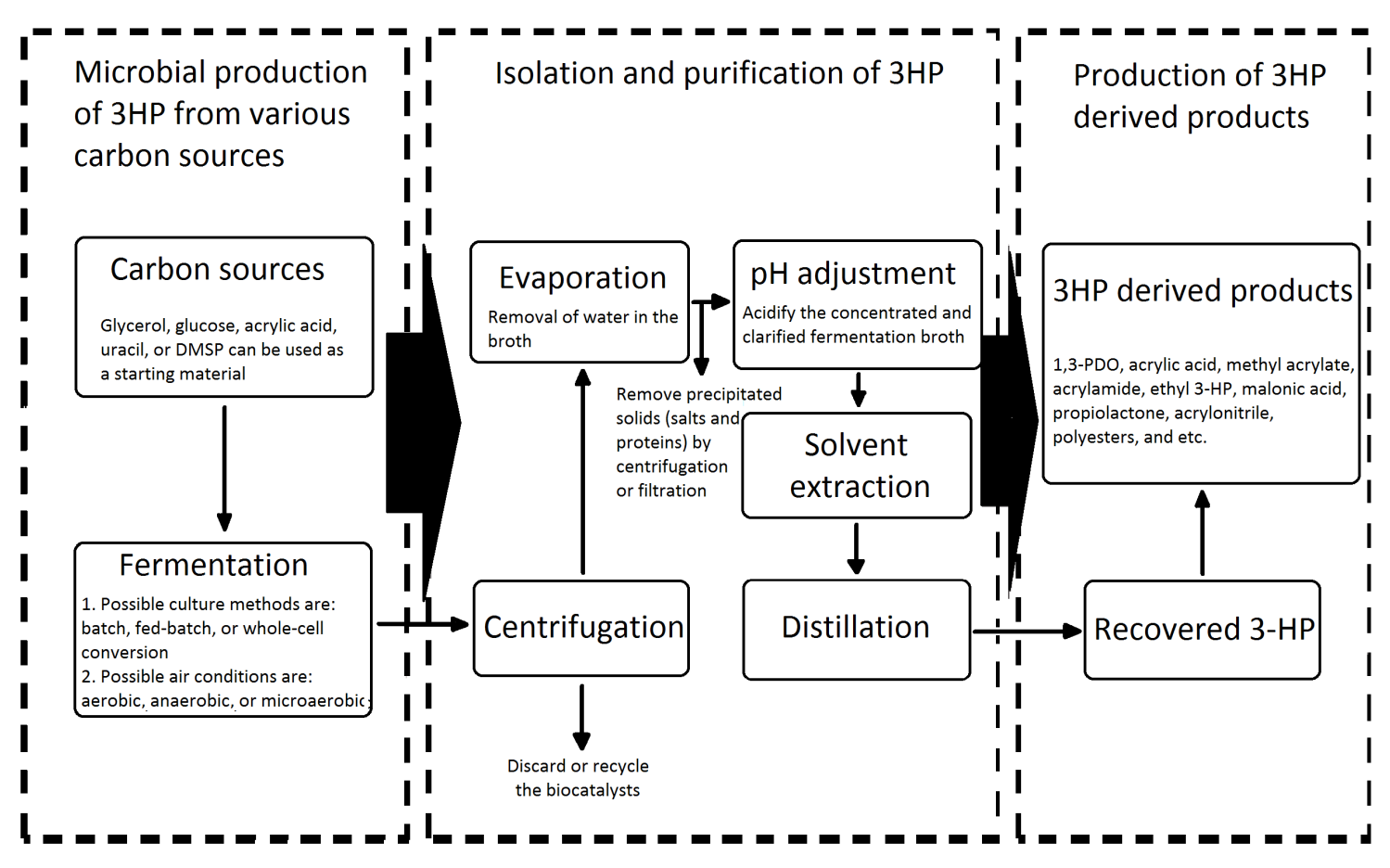

Fig. 5 Schematic illustration of 3-HP fermentation process [43]

without $g l p \mathrm{~K}$ and $y q h \mathrm{D}$, and expressing Lactobacillus brevis DhaB-DhaR, and P. aeruginosa PSALDH resulted in $57.3 \mathrm{~g} / \mathrm{L} 3$-HP concentration, $1.59 \mathrm{~g}^{*} \mathrm{~L}^{-1} * \mathrm{~h}^{-1}$ productivity and $0.88 \mathrm{~g}^{*} \mathrm{~g}^{-1}$ yield from $65 \mathrm{~g}^{*} \mathrm{~L}^{-1}$ glycerol [15].

\subsection{Highest 3-HP titer}

The highest 3-HP titer in Table 2 is $184,7 \mathrm{~g}^{*} \mathrm{~L}^{-1}$ which was produced from 3-hydroxypropionitrile (3-HPN) with immobilized cells [31]. In the experiments Escherichia coli BL21 was transformed with plasmid pET-32a-Nit190. Nit190 gene encoding a nitrilase which would be a suitable biocatalyst used to develop a highly efficient bioprocess for synthesis of 3-HP from 3-HPN. The cells were immobilized by using calcium alginate embedding and chemically crosslinking immobilized cells. Batch reactions were performed in distilled water containing $3.0 \mathrm{~mol}^{*} \mathrm{~L}^{-1}$ of substrate at the optimal conditions over $5 \mathrm{~h}$ for each batch. After each reaction, the beads were washed by distilled water and transferred to fresh reaction mixture. Repeated batch reaction was carried out under the same conditions as the first cycle. The titer $\left(184.7 \mathrm{~g}^{*} \mathrm{~L}^{-1}\right)$ and productivity $\left(36.9 \mathrm{~g}^{*} \mathrm{~L}^{-1} * \mathrm{~h}^{-1}\right)$ were obtained by isolation and purification of 3-HP from the first 30 batches [31]. Even though it is the highest titer found in the literature, the fact that only $10 \mathrm{~mL}$ was experimented makes no industrially usable technique. Further studies are needed to examine the scale-up.

The second highest 3-HP titer in Table 2 is $83,8 \mathrm{~g}^{*} \mathrm{~L}^{-1}$ converted from glycerol. The puuC(aldehyde dehydrogenase) gene was PCR-amplified from the genomic DNA of $K$. pneumoniae. The puuC gene was cloned into pTAC and pLAC, resulting in two recombinant vectors pTAC-puuC(harnessing tac promoter) and pLAC-puиC (harboring lac promoter), respectively. Transforming vectors into $K$. pneumoniae led to recombinant strains $K$. pneumoniae (pTAC-puuC) and K. pneumoniae (pLAC-puиC). $50 \mu \mathrm{g}^{*} \mathrm{~mL}^{-1}$ kanamycin was added to the medium for $K$. pneumoniae (pTAC-puuC), while $60 \mu \mathrm{g}^{*} \mathrm{~mL}^{-1}$ ampicillin was added to the medium for K. pneumoniae (pLAC-puиC). The deletion of lactic acid and acetic acid synthesis genes ldh1(lactate dehydrogenase 1), ldh2(lactate dehydrogenase 2), and pta(phosphate acetyltransferase) was carried out according to the protocol of RecA homologous recombination. Fed-batch cultivation of the strain $K$. pneumoniae $\Delta l d h 1 \Delta l d h 2 \Delta$ pta(pTAC-puuC) was carried out in 5 -L bioreactor containing $3 \mathrm{~L}$ fermentation medium. The strain was pre-cultivated in $100 \mathrm{~mL}$ fermentation medium overnight at $37^{\circ} \mathrm{C}$ and then added into the bioreactor. The agitation speed was $400 \mathrm{rpm}$ and the air was supplied at $1.5 \mathrm{vvm}$. The temperature was $37^{\circ} \mathrm{C}$ and $\mathrm{pH}$ value was adjusted to 7.0 by addition of $5 \mathrm{M} \mathrm{NaOH}$. Residual glycerol was maintained at $25 \mathrm{~g}^{*} \mathrm{~L}^{-1}$. The fermentation resulted $83.8 \mathrm{~g}^{*} \mathrm{~L}^{-1} 3-\mathrm{HP}$ in $72 \mathrm{~h}$. In the available literature this is currently the highest 3 -HP titer with $K$. pneumoniae [17].

\subsection{Highest 3-HP productivity}

The highest productivity in Table 2 is $36.9 \mathrm{~g}^{*} \mathrm{~L}^{-1} * \mathrm{~h}^{-1}$ and was already presented in detail in Section 4.2 [31].

The second highest productivity of 3 -HP in Table 2 is $1.8 \mathrm{~g}^{*} \mathrm{~L}^{-1 *} \mathrm{~h}^{-1}$ [20]. In the study a novel aldehyde dehydrogenase, GabD4 from Cupriavidus necator was identified and found that it possessed the highest enzyme activity on 3-HPA reported to 
date. The expression plasmids contained $d h a B, g d r A B$ and one of the candidate aldehyde dehydrogenase genes. This was transformed into E.coli $\triangle a c k A$-pta $\Delta y q h D$ mutant strains. The strain was cultured at $\mathrm{pH} 7.0$ in a $5 \mathrm{~L}$ bioreactor with a working volume of $2 \mathrm{~L}$ at $35^{\circ} \mathrm{C}$ and $500 \mathrm{rpm}$. The initial glycerol concentration was $80 \mathrm{~g}^{*} \mathrm{~L}^{-1}$ and concentrated glycerol $\left(700 \mathrm{~g}^{*} \mathrm{~L}^{-1}\right)$ was added to the culture broth as a feeding substrate to maintain the glycerol concentration. The recombinant E. coli produced $71.9 \mathrm{~g}^{*} \mathrm{~L}^{-1}$ of 3 -HP with a productivity of $1.8 \mathrm{~g}^{*} \mathrm{~L}^{-1} * \mathrm{~h}^{-1}[20]$.

The third highest productivity of 3 -HP in Table 2 is $1.75 \mathrm{~g}^{*} \mathrm{~L}^{-1 *} \mathrm{~h}^{-1}$ [33]. The co-production of 3-HP and 1,3-propanediol by Klebsiella pneumoniae expressing aldH was experimented under microaerobic conditions. K. pneumoniael pUC18kan-aldHec expressed aldH encoding the c-glutamyl-c-aminobutyraldehyde dehydrogenase (EC:1.2.1.19) from $E$. coli $\mathrm{K} 12$. The inoculum was prepared in $250 \mathrm{~mL}$ flask containing $100 \mathrm{~mL}$ of the preculture medium supplemented with $20 \mu \mathrm{g}^{*} \mathrm{~mL}^{-1}$ of kanamycin. The flask was inoculated with $1 \mathrm{~mL}$ of the stock culture and was incubated aerobically at $37^{\circ} \mathrm{C}$ and $220 \mathrm{rpm}$ for $12 \mathrm{~h}$. The preculture was used to inoculate $2.8 \mathrm{~L}$ of the fermentation medium in a 5-L bioreactor. The fed-batch experiments were carried out at $37^{\circ} \mathrm{C}$, and the $\mathrm{pH}$ was controlled automatically at $6.8-7.0$ with $5 \mathrm{M} \mathrm{NaOH}$. Sterile air was sparged at $1.5 \mathrm{vvm}$ with an agitation at $400 \mathrm{rpm}$. A feed of glycerol solution $\left(1.26 \times 103 \mathrm{~g}^{*} \mathrm{~L}^{-1}\right)$ was added through a peristaltic pump to maintain the concentration between 10 and $20 \mathrm{~g}^{*} \mathrm{~L}^{-1}$. When the fermentation was close to termination, feeding of glycerol was stopped to exhaust the residual glycerol. K. pneumoniae/pUC18-kan-aldHec after $28 \mathrm{~h}$ produced $48.9 \mathrm{~g}^{*} \mathrm{~L}^{-1}$ of $3-\mathrm{HP}$ and $25.3 \mathrm{~g}^{*} \mathrm{~L}^{-1}$ of 1,3-propanediol with an overall yield of 3-HP plus 1,3-propanediol of $0.66 \mathrm{~mol}^{*} \mathrm{~mol}^{-1}$ [33].

\section{Recovery}

3-Hydroxypropionic acid in particular is an important material that is useful for many industrial applications. It has been discovered that efficient recovery of $3 \mathrm{HP}$ in desired concentrations and desired purity is challenging. The recovery of 3-hydroxypropionic acid from real fermentation broth is developed and patented by Cargill [40]. Besides that, extraction experimental results were also reported [40, 41].

There was investigation about the reactive extraction of 3-HP from model aqueous solution and real bioconversion media as well [42]. In the study tri-n-octylamine (TOA) and Aliquat 336 was used as extractants in n-decanol. When 3-HP was extracted from a real bioconversion broth, some cell-originating compounds were suspected to adsorb to the aqueous-organic interface. This effect, added to the possible competition between 3-HP and compounds that can react with the extractants, led to limitations in the yield and selectivity of the extraction. For example, yield and selectivity for the model bioconversion broth were $89 \%$ and 35.9 respectively. These values decreased to $62 \%$ and 15.7 for the real broth at $\mathrm{pH}=3.2$ [42].
There was also investigation about reactive extraction of bio-based 3-HP assisted by hollow-fiber membrane contactor (HFMC) using TOA and Aliquat 336 as extractants in n-decanol [41]. The highest distribution coefficient of 3-HP $(\mathrm{KD}=13.0)$ was observed at $\mathrm{pH}=3$ with $10 \% \mathrm{v} / \mathrm{v}$ TOA mixed with $10 \% \mathrm{v} / \mathrm{v}$ Aliquat336 in n-decanol. This study showed that the acid concentration and the ratio of used amines to TOA and Aliquat 336 in n-decanol did not have a great impact on the KD during 3-HP reactive extraction, whereas it had an influence on the extraction kinetics. Moreover, 3-HP reactive extraction assisted by HFMC from real bioconversion broth with glycerol as substrate was shown to be feasible, with the same order of magnitude of extraction kinetics but with a lower KD compared with reactive extraction using model broth. The highest yield in the experiments reached $>90 \%$ [41].

The newest 3-HP recovery method from fermentation broth is patented by Cargill [40] (Fig. 5). The method comprises the steps of:

\section{- Centrifugation}

- Increasing the concentration of 3-hydroxypropionic acid and/or salts thereof in the fermentation broth by evaporation

- Acidifying the fermentation broth to lower the $\mathrm{pH}$ between 1 and 3

- Reducing the total sulfate ion and phosphate ion concentration with ion exchanger

- Extraction

- Distilling by applying vacuum and heat

\section{Conclusions and future prospects}

3-hydroxypropionic acid is a commercially valuable, important platform chemical. Several microorganisms can produce through a range of metabolic pathways. It is indispensable for the commercial production of 3-HP to use cheap and abundant substrates and also to utilize is highly efficient processes that can result a high yield, titer and productivity. Because of the fact, that natural microorganism do not perform these conditions, metabolic engineering and genetically engineered microorganism are widely studied. Several metabolic pathways are developed to utilize glucose or glycerol to produce 3-HP. Only few of them are suitable to be used economically. Using crude glycerol and convert it to 3-HP, would reduce the production costs, advancing the economic feasibility of this production. However, the many impurities found in crude glycerol, such as alcohols, salts, heavy metals, and soaps, limit its use as a substrate. Efficient production of 3-HP from glycerol with recombinant strains could be achieved by the deletion of competing pathways, in order to drive carbon flux to the reductive glycerol consumption pathway. The balance between the oxidative and reductive pathways should also be taken into consideration for effective 3-HP synthesis. The development of recombinant strains with 
native $B_{12}$ synthesis and reactivating factors would address the cofactor dependence of glycerol reduction and instability of glycerol and diol dehydratase. The development of novel and mutated ALDHs, which would have high activity on 3-HPA, would prevent the accumulation of toxic 3-HPA in the host cells, which may allow us to obtain highly efficient and productive host strains in order to produce 3-HP from glycerol. Furthermore, novel pathways for the 3-HP production from glucose can be achieved by heterologous expression of different genes and by using novel enzymes with increased specificity for the target compound. Although several pathways have already been patented for this, they are still far from potential commercial application. There still remain some important issues requiring further investigation. For example, 3-HP tolerance in 3-HP-producing strains should be further improved to enhance its titer in culture broth. With glycerol as substrate, synthesis of vitamin $\mathrm{B}_{12}$, regeneration of $\mathrm{NAD}^{+}$, and 3-HPA accumulation should be carefully studied individually or in an integrated manner. Biologically produced 3-HP results from the recent years are summarized in the Table 2 . Remarkable yields, titers, and productivities are reached by different methods in laboratory scale. Finally, an efficient and economical downstream process for separation and purification of 3-HP from the culture broth will be important as well. It has been discovered that efficient recovery of 3-HP in high concentrations and desired purity which are required for certain industrial applications is challenging. One promising solution can be the patented 3-HP recovery by Cargill $[8,10,43]$.

\section{References}

[1] Kildegaard, K. R., Jensen, N. B., Schneider, K., Czarnotta, E., Özdemir, E., Klein, T., Borodina, I. "Engineering and systems-level analysis of Saccharomyces cerevisiae for production of 3-hydroxypropionic acid via malonyl-CoA reductase-dependent pathway." Microbial Cell Factories. 15(1), pp. 53. 2016. https://doi.org/10.1186/s12934-016-0451-5

[2] Kumar, V., Ashok, S., Park, S. "Recent advances in biological production of 3-hydroxypropionic acid." Biotechnology Advances. 31(6), pp. 945-961. 2013. https://doi.org/10.1016/j.biotechadv.2013.02.008

[3] Liu, C., Ding, Y., Xian, M., Liu, M., Liu, H., Ma, Q., Zhao, G. "MalonylCoA pathway: a promising route for 3-hydroxypropionate biosynthesis." Critical Reviews in Biotechnology. pp. 1-9. 2017. https://doi.org/10.1080/07388551.2016.1272093

[4] Raj, S. M., Rathnasingh, C., Jo, J. E., Park, S. "Production of 3-hydroxypropionic acid from glycerol by a novel recombinant Escherichia coli BL21 strain." Process Biochemistry. 43(12), pp. 1440-1446. 2008. https://doi.org/10.1016/j.procbio.2008.04.027

[5] Rathnasingh, C., Raj, S. M., Jo, J. E., Park, S. "Development and evaluation of efficient recombinant Escherichia coli strains for the production of 3-hydroxypropionic acid from glycerol." Biotechnology and Bioengineering. 104(4), pp. 729-739. 2009. https://doi.org/10.1002/bit.22429

[6] Jiang, X., Meng, X., Xian, M. "Biosynthetic pathways for 3-hydroxypropionic acid production." Applied Microbiology and Biotechnology. 82(6), pp. 995-1003. 2009. https://doi.org/10.1007/s00253-009-1898-7
[7] Matsakas, L., Topakas, E., Christakopoulos, P. "New trends in microbial production of 3-hydroxypropionic acid." Current Biochemical Engineering. 1(2), pp. 141-154. 2014.

https://doi.org/10.2174/2212711901666140415200133

[8] Tingirikari, J. M. R., Ahmed, S., Yata, V. K. "Chapter 6-3-Hydroxy-propionic Acid." In: Brar, S. K., Sarma, S. J., Pakshirajan, K. (eds.) Platform Chemical Biorefinery. (pp. 101-117.), Elsevier, Amsterdam, 2016. https://doi.org/10.1016/B978-0-12-802980-0.00006-7

[9] Lee, S. H., Park, S. J., Park, O. J., Chao, J., Rhee, J. W. "Production of 3-hyroxypropionic acid from acrylic acid by newly isolated Rhodococcus erythropolis LG12." Journal of Microbiology and Biotechnology. 19(5), pp. 474-481. 2009. https://doi.org/10.4014/jmb.0808.473

[10] Kumar, V, Ashok, S., Park, S "Microbial Production of 3-Hydroxypropionic Acid From Renewable Sources: A Green Approach as an Alternative to Conventional Chemistry." In: Bisaria, V. S., Kondo, A. (eds.) Bioprocessing of Renewable Resources to Commodity Bioproducts. (pp. 381-407.), John Wiley \& Sons, Inc., 2014. https://doi.org/10.1002/9781118845394.ch14

[11] Berg, I. A., Kockelkorn, D., Ramos-Vera, W. H., Say, R. F., Zarzycki, J., Hügler, M., Alber, B. E., Fuchs, G. "Autotrophic carbon fixation in archaea." Nature Reviews Microbiology. 8(6), pp. 447-60. 2010. https://doi.org/10.1038/nrmicro2365

[12] Schwarz, M., Köpcke, B., Weber, R. W. S., Sterner, O., Anke, H. "3-Hydroxypropionic acid as a nematicidal principle in endophytic fungi." Phytochemistry. 65(15), pp. 2239-2245. 2004. https://doi.org/10.1016/j.phytochem.2004.06.035

[13] Jarboe, L. R., Zhang, X., Wang, X., Moore, J. C., Shanmugam, K. T., Ingram, L. O. "Metabolic engineering for production of biorenewable fuels and chemicals: Contributions of synthetic biology." Journal of Biomedicine and Biotechnology. 2010, Article ID 761042. 2010. https://doi.org/10.1155/2010/761042

[14] Tsuruno, K., Honjo, H., Hanai, T. "Enhancement of 3-hydroxypropionic acid production from glycerol by using a metabolic toggle switch." Microbial Cell Factories. 14(1), pp. 155. 2015. https://doi.org/10.1186/s12934-015-0342-1

[15] Kim, K., Kim, S. K., Park, Y. C., Seo, J. H. "Enhanced production of 3-hydroxypropionic acid from glycerol by modulation of glycerol metabolism in recombinant Escherichia coli." Bioresource Technology. 156, pp. 170-175. 2014. https://doi.org/10.1016/j.biortech.2014.01.009

[16] Ko, Y., Ashok, S., Zhou, S., Kumar, V., Park, S. "Aldehyde dehydrogenase activity is important to the production of 3-hydroxypropionic acid from glycerol by recombinant Klebsiella pneumoniae." Process Biochemistry. 47(7), pp. 1135-1143. 2012.

https://doi.org/10.1016/j.procbio.2012.04.007

[17] Li, Y., Wang, X., Ge, X., Tian, P. "High Production of 3-Hydroxypropionic Acid in Klebsiella pneumoniae by Systematic Optimization of Glycerol Metabolism." Scientific Reports. 6(26932), pp. 10. 2016. https://doi.org/10.1038/srep26932

[18] Lynch, M. D., Gill, R. T., Warnecke-Lipscomb, T. "Method for producing 3-hydroxypropionic acid and other products." European Patent Office PCT/US2010/050436. 2011.

[19] Kildegaard, K. R., Wang, Z., Chen, Y., Nielsen, J., Borodina, I. "Production of 3-hydroxypropionic acid from glucose and xylose by metabolically engineered Saccharomyces cerevisiae." Metabolic Engineering Communications. 2, pp. 132-136. 2015. https://doi.org/10.1016/j.meteno.2015.10.001

[20] Chu, H. S., Kim, Y. S., Lee, C. M., Lee, J. H., Jung, W. S., Ahn, J. H., Cho, K. M. "Metabolic engineering of 3-hydroxypropionic acid biosynthesis in Escherichia coli." Biotechnology and Bioengineering. 112(2), pp. 356-364. 2015. https://doi.org/10.1002/bit.25444 
[21] Jiang, W., Wang, S., Yang, Z., Fang, B. "B 12 -independent glycerol dehydratase and its reactivase from Clostridia butyricum: Optimizing cloning by uniform design logic." Engineering in Life Sciences. 15(5), pp. 519-524. 2015. https://doi.org/10.1002/elsc.201400217

[22] Brien, J. R. O., Raynaud, C., Croux, C., Girbal, L., Soucaille, P., Lanzilotta, W. N., Uni, V. "Insight into the Mechanism of the $\mathrm{B}_{12}$ -Independent Glycerol Dehydratase from Clostridium butyricum: Preliminary Biochemical and Structural Characterization." Biochemistry. 43(16), pp. 4635-4645. 2004. https://doi.org/10.1021/bi035930k

[23] Li, J., Zong, H., Zhuge, B., Lu, X., Fang, H., Sun, J. "Immobilization of Acetobacter sp. CGMCC 8142 for efficient biocatalysis of 1,3-propanediol to 3-hydroxypropionic acid." Biotechnology and Bioprocess Engineering. 21(4), pp. 523-530. 2016. https://doi.org/10.1007/s12257-016-0022-y

[24] Chen, Z., Huang, J., Wu, Y., Liu, D. "Metabolic engineering of Corynebacterium glutamicum for the production of 3-hydroxypropionic acid from glucose and xylose." Metabolic Engineering. 39(2017), pp. 151-158. 2016. https://doi.org/10.1016/j.ymben.2016.11.009

[25] Kwak, S., Park, Y. C., Seo, J. H. "Biosynthesis of 3-hydroxypropionic acid from glycerol in recombinant Escherichia coli expressing Lactobacillus brevis dhaB and dhaR gene clusters and E. coli K-12 aldH." Bioresource Technology. 135, pp. 432-439. 2013.

https://doi.org/10.1016/j.biortech.2012.11.063

[26] Honjo, H., Tsuruno, K., Tatsuke, T., Sato, M., Hanai, T. "Dual synthetic pathway for 3-hydroxypropionic acid production in engineered Escherichia coli." Journal of Bioscience and Bioengineering. 120(2), pp. 199-204. 2015. https://doi.org/10.1016/j.jbiosc.2014.12.023

[27] Mohan Raj, S., Rathnasingh, C., Jung, W.-C., Park, S. "Effect of process parameters on 3-hydroxypropionic acid production from glycerol using a recombinant Escherichia coli." Applied Microbiology and Biotechnology. 84(4), pp. 649-57. 2009.

https://doi.org/10.1007/s00253-009-1986-8

[28] Jung, W. S., Kang, J. H., Chu, H. S., Choi, I. S., Cho, K. M. "Elevated production of 3-hydroxypropionic acid by metabolic engineering of the glycerol metabolism in Escherichia coli." Metabolic Engineering. 23, pp. 116-122. 2014. https://doi.org/10.1016/j.ymben.2014.03.001

[29] Rathnasingh, C., Raj, S. M., Lee, Y., Catherine, C., Ashok, S., Park, S. "Production of 3-hydroxypropionic acid via malonyl-CoA pathway using recombinant Escherichia coli strains." Journal of Biotechnology. 157(4), pp. 633-640. 2012. https://doi.org/10.1016/j.jbiotec.2011.06.008

[30] Jung, I. Y., Lee, J. W., Min, W. K., Park, Y. C., Seo, J. H. "Simultaneous conversion of glucose and xylose to 3-hydroxypropionic acid in engineered Escherichia coli by modulation of sugar transport and glycerol synthesis." Bioresource Technology. 198, pp. 709-716. 2015. https://doi.org/10.1016/j.biortech.2015.09.079

[31] Yu, S., Yao, P., Li, J., Ren, J., Yuan, J., Feng, J., Zhu, D. "Enzymatic synthesis of 3-hydroxypropionic acid at high productivity by using free or immobilized cells of recombinant Escherichia coli." Journal of Molecular Catalysis B: Enzymatic. 129, pp. 37-42. 2016. https://doi.org/10.1016/j.molcatb.2016.03.011

[32] Sankaranarayanan, M., Ashok, S., Park, S. "Production of 3-hydroxypropionic acid from glycerol by acid tolerant Escherichia coli." Journal of Industrial Microbiology and Biotechnology. 41(7), pp. 1039-1050. 2014. https://doi.org/10.1007/s10295-014-1451-2

[33] Huang, Y., Li, Z., Shimizu, K., Ye, Q. "Co-production of 3-hydroxypropionic acid and 1,3-propanediol by Klebseilla pneumoniae expressing ald $\mathrm{H}$ under microaerobic conditions." Bioresource Technology. 128, pp. 505-512. 2013. https://doi.org/10.1016/j.biortech.2012.10.143
[34] Ashok, S., Mohan Raj, S., Ko, Y., Sankaranarayanan, M., Zhou, S., Kumar, V., Park, S. "Effect of puuC overexpression and nitrate addition on glycerol metabolism and anaerobic 3-hydroxypropionic acid production in recombinant Klebsiella pneumoniae $\Delta g l p K \Delta d h a T$." Metabolic Engineering. 15(2013), pp. 10-24. 2013.

https://doi.org/10.1016/j.ymben.2012.09.004

[35] Luo, L. H., Kim, C. H., Heo, S. Y., Oh, B. R., Hong, W. K., Kim, S., Seo, J. W. "Production of 3-hydroxypropionic acid through propionaldehyde dehydrogenase PduP mediated biosynthetic pathway in Klebsiella pneumoniae." Bioresource Technology. 103(1), pp. 1-6. 2012. https://doi.org/10.1016/j.biortech.2011.09.099

[36] Kumar, V., Sankaranarayanan, M., Durgapal, M., Zhou, S., Ko, Y., Ashok, S., Park, S. "Simultaneous production of 3-hydroxypropionic acid and 1,3-propanediol from glycerol using resting cells of the lactate dehydrogenase-deficient recombinant Klebsiella pneumoniae overexpressing an aldehyde dehydrogenase." Bioresource Technology. 135, pp. 555-563. 2013. https://doi.org/10.1016/j.biortech.2012.11.018

[37] Wang, Y., Sun, T., Gao, X., Shi, M., Wu, L., Chen, L., Zhang, W. "Biosynthesis of platform chemical 3-hydroxypropionic acid (3-HP) directly from CO2 in cyanobacterium Synechocystis sp. PCC 6803." Metabolic Engineering. 34, pp. 60-70. 2016. https://doi.org/10.1016/j.ymben.2015.10.008

[38] Chen, Y., Bao, J., Kim, I. K., Siewers, V., Nielsen, J. "Coupled incremental precursor and co-factor supply improves 3-hydroxypropionic acid production in Saccharomyces cerevisiae." Metabolic Engineering. 22, pp. 104-109. 2014. https://doi.org/10.1016/j.ymben.2014.01.005

[39] Borodina, I., Kildegaard, K. R., Jensen, N. B., Blicher, T. H., Maury, J., Sherstyk, S., Nielsen, J. "Establishing a synthetic pathway for high-level production of 3-hydroxypropionic acid in Saccharomyces cerevisiae via $\beta$-alanine." Metabolic Engineering. 27(2015), pp. 57-64. 2015. https://doi.org/10.1016/j.ymben.2014.10.003

[40] Abraham, T. W., Allen, E., Hahn, J. J., Tsobanakis, P., Bohnert, E. C., Frank, C. L. "Recovery of 3-hydroxypropionic acid." European Patent Office PCT/US2014/028793. 2014.

[41] Burgè, G., Chemarin, F., Moussa, M., Saulou-Bèrion, C., Allais, F., Spinnler, H.-É., Athès, V. "Reactive extraction of bio-based 3-hydroxypropionic acid assisted by hollow-fiber membrane contactor using TOA and Aliquat 336 in n-decanol." Journal of Chemical Technology and Biotechnology. 91(10), pp. 2705-2712. 2016. https://doi.org/10.1002/jctb.4878

[42] Moussa, M., Burgé, G., Chemarin, F., Bounader, R., Saulou-Bérion, C., Allais, F., Athès, V. "Reactive extraction of 3-hydroxypropionic acid from model aqueous solutions and real bioconversion media. Comparison with its isomer 2-hydroxypropionic (lactic) acid." Journal of Chemical Technology and Biotechnology. 91(8), pp. 2276-2285. 2016. https://doi.org/10.1002/jctb.4813

[43] Yokimiko, D., Young, H. O., Mary Grace, B., Kei-Anne, B., Jeong Chan, J., Cheol Gi, C., Si Jae, P. "Microbial Production of 3-Hydroxypropionic Acid." In: Wittmann, C., Liao, J. C. (eds.), Industrial Biotechnology Products and Processes. (pp. 411-435), Wiley, 2017. https://doi.org/10.1002/9783527807833.ch14 